\title{
Multiple measures of thermal performance of early stage eastern rock lobster in a fast-warming ocean region
}

\author{
Samantha Twiname ${ }^{1, *}$, Quinn P. Fitzgibbon ${ }^{1}$, Alistair J. Hobday ${ }^{2}$, Chris G. Carter ${ }^{1}$, \\ Gretta T. Pecl ${ }^{1}$
}

${ }^{1}$ Institute for Marine and Antarctic Studies, University of Tasmania, Private Bag 49, Hobart, TAS 7001, Australia ${ }^{2}$ CSIRO Oceans and Atmosphere, Castray Esplanade, Hobart, TAS 7001, Australia

\begin{abstract}
To date, many studies trying to understand species' climate-driven changes in distribution, or 'range shifts', have each focused on a single potential mechanism. While a single performance measure may give some insight, it may not be enough to accurately predict outcomes. Here, we used multiple measures of performance to explore potential mechanisms behind species range shifts. We examined the thermal pattern for multiple measures of performance, including measures of aerobic metabolism and multiple aspects of escape speed, using the final larval stage (puerulus) of eastern rock lobster Sagmariasus verreauxi as a model species. We found that aerobic scope and escape speed had different thermal performances and optimal temperatures. The optimal temperature for aerobic scope was $27.5^{\circ} \mathrm{C}$, while the pseudo-optimal temperature for maximum escape speed was $23.2^{\circ} \mathrm{C}$. This discrepancy in thermal performance indicators illustrates that one measure of performance may not be sufficient to accurately predict whole-animal performance under future warming. Using multiple measures of performance and appropriate modelling techniques may lead to a more accurate prediction of future range shifts, including the timing and extent of climate-driven species redistribution.
\end{abstract}

KEY WORDS: Aerobic scope - Climate change · Crustacean larvae $\cdot$ Escape speed $\cdot$ Puerulus $\cdot$ Respiratory metabolism $\cdot$ Sagmariasus verreauxi

\footnotetext{
${ }^{*}$ Corresponding author: samantha.twiname@utas.edu.au
}

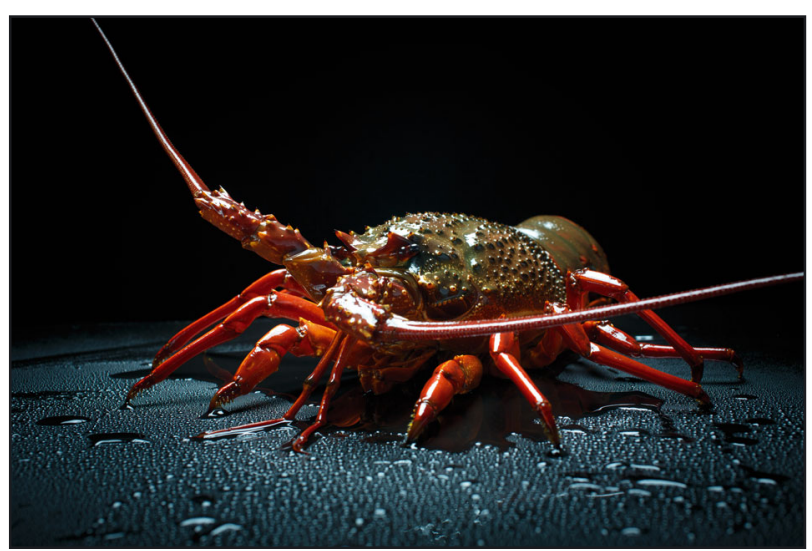

Single thermal performance measures for eastern rock lobster Sagmariasus verreauxi may not accurately predict whole animal performance under future ocean warming.

Photo: Peter Mathew

\section{INTRODUCTION}

Ocean warming is affecting marine species worldwide (Pecl et al. 2017). One of the most prevalent effects are changes to species' geographical distributions (Sunday et al. 2012, Poloczanska et al. 2013). While these 'range shifts' occur in many marine systems, we do not yet have a clear understanding of the mechanisms driving the high variation in rate and magnitude of the changes within (Last et al. 2011, Sunday et al. 2015) and between regions (Poloczan-

() The authors 2019. Open Access under Creative Commons by Attribution Licence. Use, distribution and reproduction are unrestricted. Authors and original publication must be credited. 
ska et al. 2013). Part of the explanation may lie in the complex physiology of marine species and how each aspect of their physiology reacts to changes in their thermal environments.

Many marine species are ectothermic, meaning that their internal physiology is highly dependent on ambient temperature which, in turn, strongly influences a species' function and behaviour (Pörtner \& Knust 2007, Pörtner \& Farrell 2008, Doney et al. 2012). To understand the effect of temperature on many physiological and biological processes, thermal performance curves can be constructed. Thermal performance curves allow visualisation of the effects of temperature on bodily rates such as metabolism, growth and reproduction (Eme \& Bennett 2009, Dell et al. 2014, Donelson et al. 2014). With increasing ocean temperatures, bodily rates are likely to change due to heightened metabolic performance, with consequent effects on species performance and behaviour. While single thermal performance curves may provide some insight to how a particular body function may change under ocean warming, it may be of limited use as a predictor of whole-animal performance.

Aerobic scope, a proxy for the amount of energy available for non-essential body processes, is a key component of organismal energy budgets and is dependent on ambient temperature experienced by ectotherms (Watson et al. 2014). The oxygen and capacity-limited thermal tolerance hypothesis proposes that there is a limited thermal range for individual ectotherm species in which performance, measured as aerobic scope, is maximised (Pörtner \& Knust 2007). This optimisation, and subsequent decline in performance at temperatures outside of this optimal range, is hypothesised to be due to the mismatch between oxygen demand and supply (Pörtner $\&$ Knust 2007). This measure of organismal performance has been proposed as a way to predict future changes in population characteristics under climate change (Pörtner \& Farrell 2008). While aerobic scope is a valuable measure of performance, there has been an increase in the number of studies that suggest that aerobic scope is an incomplete measure of wholeorganism performance (Norin et al. 2014, Fitzgibbon et al. 2017, Jutfelt et al. 2018). Different organismal processes have different responses to increases in ambient temperatures, such as the difference between growth rate optimums and aerobic scope in juvenile eastern rock lobster Sagmariasus verreauxi (Fitzgibbon et al. 2017) and different thermal optima for feeding and growth performance in juvenile barramundi Lates calcarifer (Katersky \& Carter 2007). Moreover, aerobic scope is not useful for predicting thermal preference or performance in juvenile barramundi (Norin et al. 2014).

To gain a more robust understanding of the effects of climate change on marine communities, we need to incorporate measures of species' interactions, such as competition or predation performance, along with this individual physiological performance data (Evans et al. 2015). Another potential constraint, or conversely, facilitator, to species' range change under ocean warming are inter-specific interactions. Changes to a species' individual physiology under climate change are also likely to influence its interactions with other species, by modifying or eliminating current interactions or creating new ones (Pitt et al. 2010, Kordas et al. 2011, Pinsky et al. 2013). For example, predation plays a critical role in the structuring and functioning of marine communities, as predator-prey interactions provide a link for transfer of energy, nutrients and materials from basal species to apex predators (Paine 1974, Dell et al. 2014). Predator-prey interactions are affected by temperature through effects on active body velocity in ectotherms (Grigaltchik et al. 2012, Dell et al. 2014). As attack and escape performance are key factors in the outcome of predator-prey interactions, the effect of ocean warming on ectotherm mobility and locomotion may have far-reaching effects on marine community structure (Cairns et al. 2008, Dell et al. 2014, Öhlund et al. 2015).

The south east coast of Australia has been identified as an ocean warming hotspot, where rates of warming are up to 4 times the global average (Hobday \& Pecl 2014, Pecl et al. 2014). In recent years, Australia has seen many changes in the distribution and abundance of local species as well as new species not previously recorded in the region, from pelagic fish to sedentary invertebrates (Pitt et al. 2010, Last et al. 2011, Robinson et al. 2015). One such species suspected of undergoing a range shift into the area is the eastern rock lobster $S$. verreauxi (H. Milne Edwards, 1851), a large species of spiny lobster found along the east coast of Australia between southern Queensland and northern Tasmania, as well as New Zealand. There is evidence that $S$. verreauxi is becoming more common further south along the Tasmanian east coast (Robinson et al. 2015), although isolated individuals have been reported over many decades. This potential range shift has implications for both fisheries and ecological management. While not currently a targeted fisheries species in Tasmania due to low abundance, continued ocean warming and increased $S$. verreauxi recruitment may facilitate a new lobster fishery or compete with the more valuable existing southern rock lobster Jasus edwardsii fishery in the region. 
Spiny lobsters have among the longest and most complex larval life cycles of any marine invertebrate, lasting for up to $2 \mathrm{yr}$ in some species, with this stage being important for dispersal and possible range extension (Phillips et al. 2006). These early life stages may also be more susceptible to effects of climate change and ocean warming than their juvenile and adult counterparts (Pörtner \& Farrell 2008, Storch et al. 2011, Fitzgibbon et al. 2014b). The puerulus stage of the spiny lobster life cycle is a critical transitional stage from pelagic larvae to recruitment into the juvenile population. It is a nektonic, non-feeding stage that actively swims to settle onto suitable habitat substrate in coastal regions (Fitzgibbon et al. 2014a). Like many larvae settling into new communities, puerulus must survive the 'wall of mouths' in their new environment (Emery 1973, Hamner et al. 1988). Physiological performance, such as escape speed, is critical for survival for puerulus stage $S$. verreauxi, and changes to escape speed with warming may have far-reaching effects for the local and potentially range-shifting population.

To understand the future capacity for potential range shifts, we investigated multiple measures of $S$. verreauxi thermal performance, specifically measures of aerobic performance and escape performance. The specific aim was to develop thermal performance curves for both measures of performance and determine if thermal optimums for each measure were similar or different. Our hypotheses are, firstly, that the measures of aerobic scope and escape speed will have different thermal optimums, based on previous work on $S$. verreauxi juveniles that showed differences in other measures of performance (Fitzgibbon et al. 2017), and secondly, that escape speed will not change with temperature as it is an important survival trait that is likely strongly selected for and will hence be less affected by environmental changes.

\section{MATERIALS AND METHODS}

\subsection{Animal collection and holding}

Puerulus stage eastern rock lobster Sagmariasus verreauxi were reared from eggs at the Institute for Marine and Antarctic Studies (IMAS) aquaculture facility in Hobart, Australia, as described by Fitzgibbon \& Battaglene (2012) and Fitzgibbon et al. (2012). Fertilised eggs from different brood stock individuals were reared together, resulting in a mixed pool of larvae from which individuals for experimental trials were haphazardly selected. After metamorphosis from phyllosoma to puerulus stage larvae, animals were transferred to individual cylindrical vessels (300 ml) suspended in a 681 polypropylene sump. Each sump held 9 vessels, each with a single puerulus within, with each temperature treatment conducted in separate sumps. Each vessel had a $5 \mathrm{~mm}$ oyster mesh shelter for the pueruli, a mesh bottom and received water circulated from the sump. The sumps were supplied with flow-through filtered seawater from a larger temperature-controlled sump at a rate of $50 \mathrm{l} \mathrm{h}^{-1}\left(0.74\right.$ exchanges $\left.\mathrm{h}^{-1}\right)$. An air stone was used to increase mixing and aeration within each sump, which was maintained above $100 \%$ dissolved oxygen saturation. Upon placement of pueruli into this holding system, they were held for a period of $7-9 \mathrm{~d}$ at $21^{\circ} \mathrm{C}$ before any experimental treatments were applied. This was done to eliminate any effects of the moulting cycle on the metabolic measurements but was limited by the time period before subsequent moults. $S$. verreauxi are at the puerulus stage for approximately $21 \mathrm{~d}$, and trials were conducted from age 10-15 d, making sure that there was no effect of moulting on the trials (Fitzgibbon et al. 2014a). Animal holding was conducted under a $12 \mathrm{~h}$ light:12 h dark regime and marine seawater (33-35\%) salinity conditions.

\subsection{Experimental protocol}

Pueruli were assessed for metabolic rates and escape speed at 6 different temperature treatments: $20,22,24,26,28$ and $30^{\circ} \mathrm{C}(\mathrm{n}=6,6,6,10,10$ and 9 replicates, respectively; $0.131 \pm 0.052 \mathrm{~g}$ average wet weight). This temperature range spanned thermal regimes currently experienced by $S$. verreauxi as well as above those that may be reached with future ocean warming. From the holding temperature of $21^{\circ} \mathrm{C}$, each of the 6 temperature treatments were reached by increasing or decreasing the temperature via submersible heaters (ir33, Carel) by $2{ }^{\circ} \mathrm{C} \mathrm{d}^{-1}$ followed by a $2 \mathrm{~d}$ acclimation period. Due to different times taken to reach trial temperatures, there was an age difference in the puerulus of $5 \mathrm{~d}$ between the 20 and $30^{\circ} \mathrm{C}$ treatments. Even with the age differences, all puerulus remained within the stable intermoult period for the species and life stage (Fitzgibbon et al. 2014a).

Metabolic rates measured at each of these temperatures included standard metabolic rate (SMR), routine metabolic rate (RMR) and active metabolic rate $(\mathrm{AMR})$, with aerobic scope calculated (aerobic scope $=$ AMR - SMR) (e.g. Fitzgibbon et al. 2014b). Excess 
post-exercise oxygen consumption (EPOC) and recovery time were also measured for each individual. For the escape speed trials, maximum (burst) speed, average speed and number of escape responses were measured. Both metabolic and escape speed trials were run concurrently. Pueruli were first placed into the intermittent flow respirometry chambers for $16 \mathrm{~h}$ to calculate SMR and RMR. After this period, they were placed into the escape speed arena and manually chased by hand for an approximately $9 \mathrm{~min}$ period while being video recorded for later analysis of escape speed, before being replaced into the respirometry chambers to measure AMR, EPOC and recovery time measurements for a further $24 \mathrm{~h}$. To clarify, for each puerulus we gathered metabolic rate, escape speed and recovery data measurements.

\subsection{Respirometry}

Oxygen consumption rates of $S$. verreauxi pueruli were measured using an intermittent flow respirometry system as described by Fitzgibbon et al. (2014b). The system comprised four $19 \mathrm{ml}$ glass micro-respiration chambers (horizontal mini chambers, Loligo Systems) connected to 4 twin channel mini peristaltic pumps (Mini-Peristaltic Pump II, Harvard Apparatus). Two pumps ensured circulation within the chambers and past the dissolved oxygen sensor at all times, and provided the open-close flushing of the chambers that replenished the oxygen levels to ambient conditions in the respirometry chambers. The chambers were submerged in a $3.5 \mathrm{l}$ outer tank maintained at the trial temperature by a sump with a submersible heater supplied with flow-through filtered seawater. The chambers were connected to oxygen sensors that measured dissolved oxygen levels every $20 \mathrm{~s}$ (OXY-4 Mini multichannel fibre optic oxygen transmitter, PreSens). The system was set to a $10 \mathrm{~min}$ on, 10 min off cycle allowing for 3 metabolic oxygen consumption measurements $\mathrm{h}^{-1}$.

Pueruli were placed in their respective chambers in the afternoon and left undisturbed for a period of $16 \mathrm{~h}$. The first $2 \mathrm{~h}$ of the oxygen consumption data were discarded to allow for chamber acclimation by the pueruli, and the following $14 \mathrm{~h}$ of data used to calculate the SMR, using the average of the 5 lowest oxygen consumption values, and RMR, calculated as the average of all measures in the $14 \mathrm{~h}$ period. After this $16 \mathrm{~h}$ period, the pueruli were removed individually from their chamber at the beginning of an open cycle and placed into a $100 \mathrm{l}$ (59 cm diameter) round chase arena. The pueruli were chased for a $9 \mathrm{~min}$ period to simulate exercise to exhaustion (see below for more details on concurrently run escape speed trials). This was done sequentially, with one puerulus chased at a time. The exercised individual was then returned to its original respirometry chamber and left for a further $24 \mathrm{~h}$. This period was used to calculate the AMR (average of the 2 highest oxygen consumption values), as well as EPOC and time taken to recover to RMR. Time taken to recover was estimated when the individual's metabolic rate came back to within 2 standard deviations of pre-exercise RMR. EPOC was calculated by the area under the curve. All respirometry trials were conducted under a $24 \mathrm{~h}$ light regime to minimise activity and allow for accurate metabolic rates to be recorded.

After respirometry trials were completed, pueruli were removed from their chambers and wet mass weighed. Background respiration was measured simultaneously for each trial via 1 of the 4 chambers being left empty and subjected to the same conditions and sampling intervals as those with trial pueruli.

\subsection{Escape speed trials}

Escape speed trials were recorded using a stereo video setup with twin video cameras (HERO 4, GoPro). A stereo video system allows for highly accurate measures of distance and speed in 3dimensions to be made, irrespective of the direction of the escape response (Harvey et al. 2002). Twin GoPro cameras were housed in waterproof housings (custom housings, SeaGIS) mounted on a stainless steel frame with the cameras angled inwards $15^{\circ}$ from the perpendicular to allow sufficient overlap of both of the cameras' fields of view. The cameras were programmed to a high speed capture rate of 120 frames per second (fps) with a resolution of $720 \mathrm{p}(1280 \times 720$ pixels $)$. The high frame rate allowed for accurate body velocity measurements to be calculated. The camera setup was mounted above the $100 \mathrm{l}, 59 \mathrm{~cm}$ diameter round chase arena supplied with filtered seawater at the same temperature as used in the respirometry trial. The tank was equipped with an air stone for circulation and an external light source to provide sufficient light for filming. Upon completion of the first $16 \mathrm{~h}$ of respirometry trials, pueruli were removed from their chamber and placed in the chase arena. There, they were manually chased for a period of approximately 9 min (deemed long enough for the puerulus to reach exhaustion, as well as to coincide with the flushing period of the respirometry trials). This 
manual chasing included gently tugging with hands on the antennae and legs and gently prodding the body to illicit an escape response. The pueruli were stimulated continually during this period. Escape responses were defined as events when the pueruli responded with a tail flick response to 'escape' the stimulus. After this period, the puerulus was removed from the arena and placed immediately into its original chamber to continue the respirometry trial. Several measures of performance were taken for escape speed for the entire 9 min trial duration: (1) the maximum speed reached by each puerulus (simulated single escape response), (2) the average escape speed (average of all escape speeds measured during the 9 min interval, simulating performance of individuals undertaking multiple escape responses) and (3) the total number of escape responses exhibited by each pueruli.

\subsection{Data and statistical analysis}

All data and statistical analyses were performed using MS Excel ${ }^{\circledR}$, EventMeasure Stereo (SeaGIS software) and $\mathrm{R}$ version 3.5.1 (R Core Team 2017). Oxygen consumption rates were calculated in Excel where linear regressions were applied to raw oxygen concentrations to determine the decrease in oxygen concentration over the closed chamber period (9 min) before being transformed to an hourly consumption rate. These consumption values were corrected for background respiration and puerulus dry mass (DW), with final metabolic rates expressed as $\mathrm{mg} \mathrm{O}_{2} \mathrm{gDW}^{-1} \mathrm{~h}^{-1}$. Oxygen consumption rates were ascertained using DW, estimated as $22 \%$ of the wet weight measured, based on data from Fitzgibbon et al. (2014a). Background respiration was measured simultaneously with the trials and was accounted for by subtracting background oxygen consumption values from the oxygen consumption values of the pueruli measurements. Recovery measures were calculated as the time and magnitude taken for metabolic rates to come back within 2 standard deviations of RMR. Magnitude of EPOC was the sum of the area under the curve between measured metabolic rates after exercise minus the RMR plus 2 standard deviations. The stereo video footage was analysed using EventMeasure Stereo with distance travelled calculated in 3 dimensions $(X, Y, Z$ coordinates) while the frame rate gave the time taken to complete the escape response. Velocity (in $\mathrm{m} \mathrm{s}^{-1}$ ) was then calculated using the following adapted Pythagoras equation:
Velocity $=\frac{\sqrt{\left(X_{\text {end }}-X_{\text {start }}\right)^{2}+\left(Y_{\text {end }}-Y_{\text {start }}\right)^{2}+\left(Z_{\text {end }}-Z_{\text {start }}\right)^{2}}}{\text { Number of video frames }}$

where $X, Y$ and $Z$ are the 3-dimension coordinates separated into 'start' position and 'end' position of the escape response, and number of video frames is the number of frames taken to complete the responses from start to end frame.

Regression modelling was used to analyse relationships between measured variables and temperature. Metabolic rates, EPOC and escape responses (maximum and average speeds as well as number of escape responses) were assessed for normality using residual plots and were analysed using linear models as well as linear mixed models. Recovery time was analysed using a binomial response model. Model selection used Akaike's information criterion (AIC) to determine the best fit model. Where appropriate, optimal temperatures $\left(T_{\mathrm{opt}}\right)$ for the thermal performance curves were calculated using the first derivative of the quadratic equation from the regression analysis. Pearson's correlation tests were used to identify parameters that were significantly correlated.

\section{RESULTS}

Metabolic rates, EPOC and escape response parameters were examined with linear as well as linear mixed models; however, in all instances the simple linear models fit better than the linear mixed models as determined by AIC values (data not shown). Therefore, all regression models referred to herein are linear models.

Pueruli metabolic rates all increased significantly with temperature, with different regression exponents (Fig. 1). Regression analyses showed RMR and AMR increased exponentially with temperature, while SMR increased linearly (Fig. 1a, Table 1). Aerobic scope was best described by a quadratic regression where the measure peaked at $27.5^{\circ} \mathrm{C}$ (Fig. $1 \mathrm{~b}$, Table 1). Mortality only occurred at the higher temperatures, with 1, 1 and 3 mortalities in the 26,28 and $30^{\circ} \mathrm{C}$ treatments, respectively. These mortalities occurred during the respirometry phase after the animals were exercised. The measurements of the animals that died during the trials were excluded from all regression analyses.

Recovery rates, time and EPOC showed different responses to temperature (Fig. 2). At all temperatures, many individuals were able to recover quickly. However, at temperatures $26^{\circ} \mathrm{C}$ and above, some individuals showed large increases in both recovery time and EPOC. For recovery time, there was a clear division between individuals that recovered quickly 


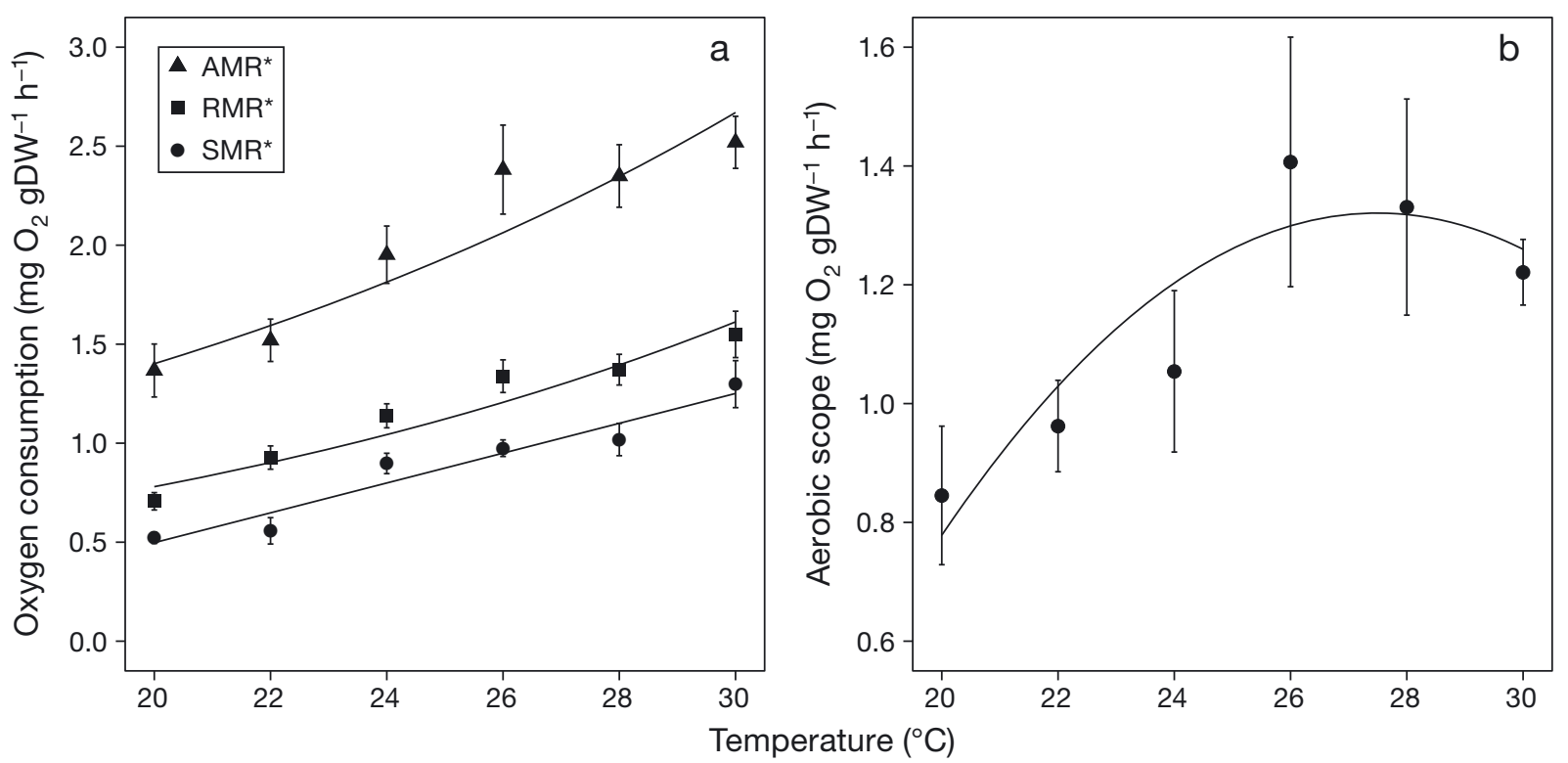

Fig. 1. Effect of temperature on aerobic metabolism of Sagmariasus verreauxi pueruli, including (a) active metabolic rate (AMR), routine metabolic rate (RMR), standard metabolic rate (SMR) and (b) aerobic scope. Values are means \pm 1 SE. Sample size ranged from 6 to 10 individuals per temperature treatment. Details for regressions are provided in Table $1 ;{ }^{*} \mathrm{p}<0.05$

Table 1. Results of regression analyses of different performance responses to temperature of Sagmariasus verreauxi pueruli. AMR: active metabolic rate; RMR: routine metabolic rate; SMR: standard metabolic rate; EPOC: excess post-exercise oxygen consumption. Analyses are in the form of linear $(y=a x+b)$, exponential $(y=\exp (a x+b))$ and quadratic $\left(y=a x^{2}+b x+c\right)$ regressions; - : parameter not included in model ${ }_{i}^{*} p<0.05$

\begin{tabular}{|c|c|c|c|c|c|c|c|c|}
\hline & Regression & $a$ & $b$ & C & $\mathrm{R}^{2}$ & df & $F$ & $\mathrm{p}$ \\
\hline AMR & Exponential & 0.065 & -0.952 & - & 0.4845 & 44 & 41.35 & $<0.001^{*}$ \\
\hline RMR & Exponential & 0.073 & -1.700 & - & 0.6325 & 44 & 75.72 & $<0.001^{*}$ \\
\hline SMR & Linear & 0.075 & -1.010 & - & 0.5863 & 44 & 62.36 & $<0.001^{*}$ \\
\hline Aerobic scope & Quadratic & -0.010 & 0.531 & -5.983 & 0.1481 & 43 & 3.738 & $0.032^{*}$ \\
\hline EPOC & Exponential & 0.542 & -0.106 & - & 0.0138 & 44 & 0.617 & 0.436 \\
\hline Maximum speed & Quadratic & -0.005 & 0.214 & -1.720 & 0.1306 & 42 & 3.154 & 0.053 \\
\hline Average speed & Quadratic & -0.002 & 0.118 & -0.926 & 0.1307 & 42 & 3.158 & 0.053 \\
\hline No. of escape responses & Quadratic & -0.149 & 7.411 & -80.008 & 0.0531 & 43 & 1.206 & 0.309 \\
\hline
\end{tabular}

and those that did not (Fig. 2a). A binomial response model showed temperature affected whether not an individual came back to its RMR quickly or not, with predicted recovery decreasing with increasing temperature (Wald test, $\chi^{2}=6.7, p=0.0096$ ). Individuals that took longer to recover had associated larger values of EPOC. However, exponential regression analysis showed there was no significant effect of temperature on EPOC measurements $\left(F_{1,44}=0.6171, \mathrm{p}=\right.$ 0. 4363; Fig. 2b).

Analysis of 2 measures of escape speed-maximum escape speed (single burst response) and average escape speed (average of all escape responses) showed both responses were non-significant across the temperature range tested $\left(F_{2,42}=3.153, \mathrm{p}=0.053\right.$ and $F_{2,42}=3.158, p=0.053$ respectively; Fig. 3a, Table 1). Though this relationship was non-significant at $p=0.053$, due to its near significant at $\alpha=0.05$ and the relationship between maximum escape speed and temperature best described by a quadratic model, a pseudo-optimal temperature was calculated, which peaked at $23.2^{\circ} \mathrm{C}$. The number of escape responses did not differ significantly with temperature (Fig. 3b, Table 1).

Results of Pearson correlation tests showed a significant positive relationship between log-transformed recovery time and log-transformed magnitude of EPOC values $\left(\mathrm{r}_{44}=0.943, \mathrm{p}<0.001\right)$. There was a weak correlation between aerobic scope and loglinear EPOC values $\left(\mathrm{r}_{44}=0.325, \mathrm{p}=0.027\right)$ but not 


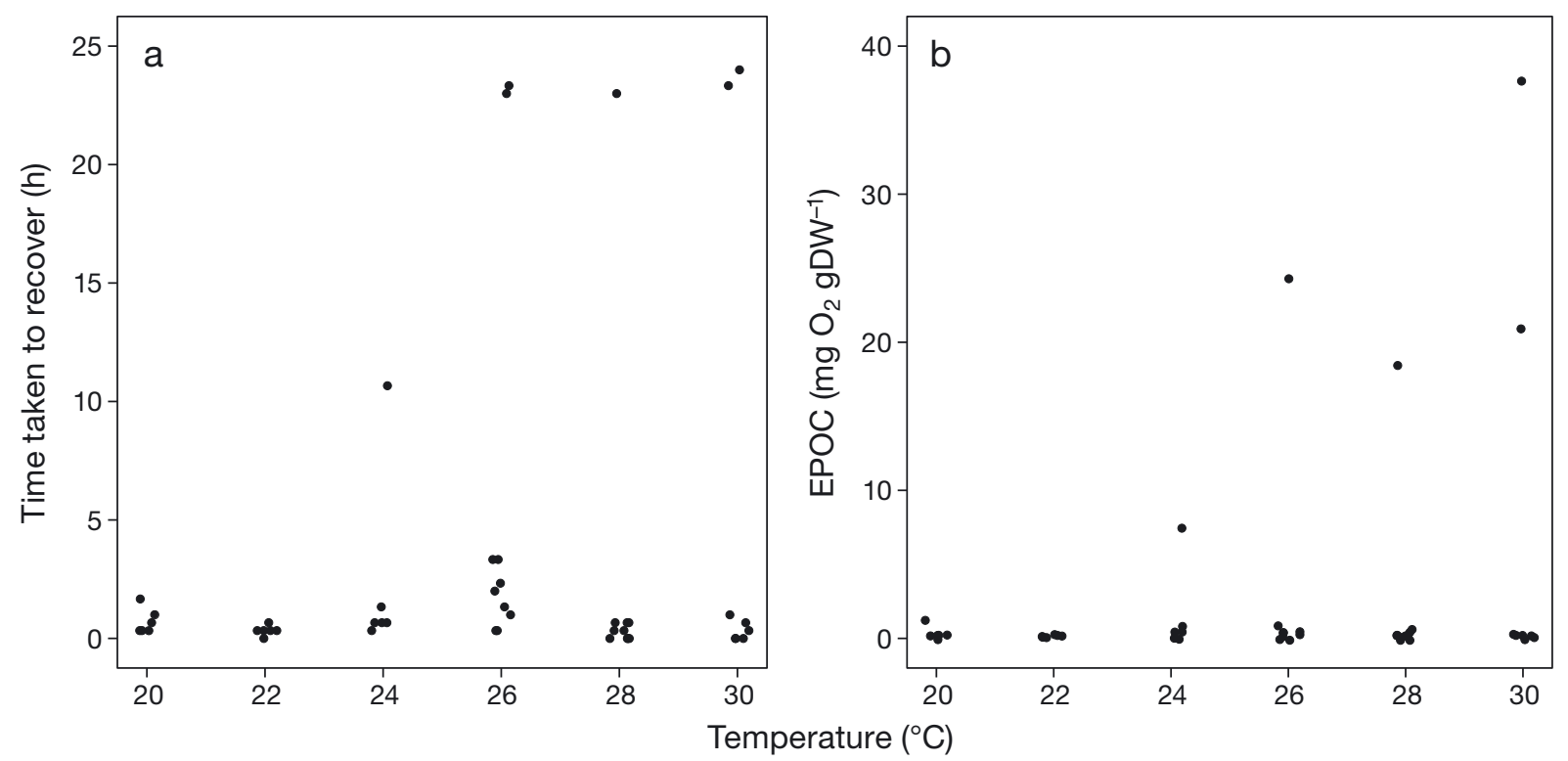

Fig. 2. Recovery measurements of Sagmariasus verreauxi pueruli including (a) recovery time data and (b) excess post-exercise oxygen consumption (EPOC). Sample size ranged from 6 to 10 individuals per temperature treatment. Details for EPOC regression are provided in Table 1

between aerobic scope and log-linear recovery times $\left(r_{44}=0.281, p=0.058\right)$. There were no significant correlations between aerobic scope and maximum escape speed $\left(\mathrm{r}_{43}=-0.116, \mathrm{p}=0.448\right)$ or between maximum escape speed and log-linear recovery measures (recovery time $\mathrm{r}_{43}=-0.007, \mathrm{p}=0.961 ;$ EPOC $\mathrm{r}_{43}=0.005$, $\mathrm{p}=0.976)$. There was a significant positive correla- tion between average escape speed and puerulus weight $\left(r_{43}=0.326, p=0.029\right)$ but not between maximum escape speed and weight $\left(\mathrm{r}_{43}=0.281, \mathrm{p}=\right.$ 0.061). Level of activity (i.e. number of escape responses; Fig. 3b) did not correlate with longer recovery times or the magnitude of EPOC $\left(\mathrm{r}_{44}=0.191, \mathrm{p}=\right.$ 0.203 and $\mathrm{r}_{44}=0.1664, \mathrm{p}=0.276$, respectively).

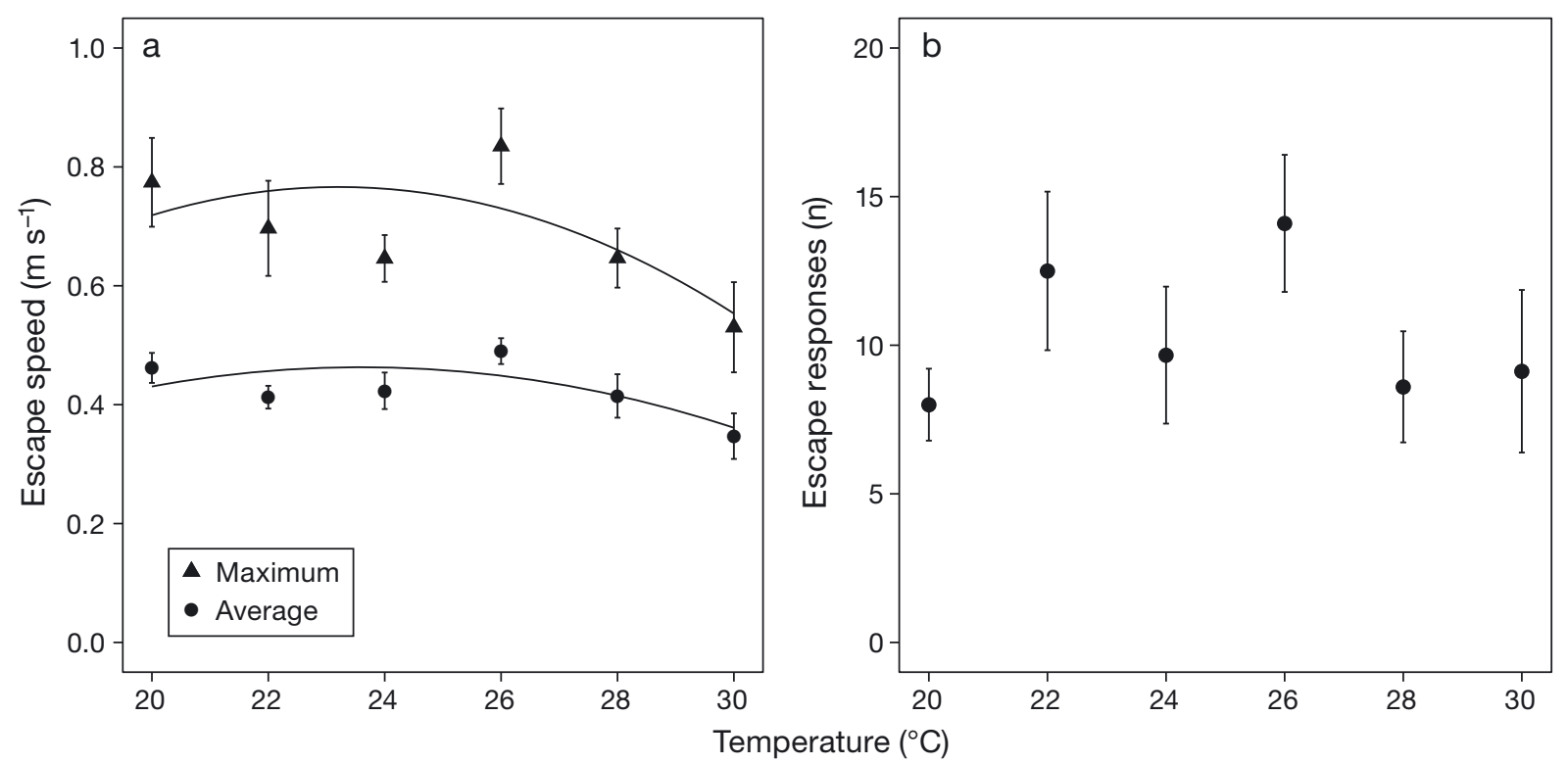

Fig. 3. (a) Maximum and average escape speeds, and (b) number of escape responses exhibited by Sagmariasus verreauxi pueruli. Values are mean $\pm 1 \mathrm{SE}$. Sample size ranged from 6 to 10 individuals per temperature treatment. Details for regressions are provided in Table 1 


\section{DISCUSSION}

Sagmariasus verreauxi pueruli exhibited different thermal optimums for multiple measures of species performance. Our optimal temperature results for aerobic scope and escape speed support increasing evidence suggesting that a single measure of performance, namely aerobic scope, may not accurately predict whole-animal performance under ocean warming scenarios (Clark et al. 2013, Norin et al. 2014, Fitzgibbon et al. 2017, Jutfelt et al. 2018).

\subsection{Differences in thermal tolerances between performance measures}

Different thermal optima of different measures of performance have been found in a range of marine species, as well as across different life stages in the same species. In juvenile $S$. verreauxi, optimal growth temperatures did not match the thermal optimum for aerobic scope (Fitzgibbon et al. 2017). This has also been reported in other species, such as barramundi Lates calcarifer, where different thermal optimums were found for juvenile feed intake and growth performance (Katersky \& Carter 2007). Aerobic scope was also not found to be a predictor of behavioural thermoregulation or performance for juveniles of the same species (Norin et al. 2014). The pseudo-optimal temperature for escape speed $\left(23.2^{\circ} \mathrm{C}\right)$ was lower than the optimal temperature for aerobic scope $\left(27.5^{\circ} \mathrm{C}\right)$, indicating that while an individual may have the aerobic capacity to survive and perform well in some areas, escape speed could potentially be a limiting factor in whole-organism performance at higher temperatures, potentially resulting in mortality due to being unable to escape a predator. Collectively, this shows that multiple performance measures can have different thermal optimums which may contribute differently to how a species copes with ocean warming.

Measures of performance may also have differing thermal optimums at different life stages. Prior studies have suggested that larval stages of marine species may be more susceptible to climate change (Pörtner \& Farrell 2008). While there have been studies on aerobic scope in juvenile stages of $S$. verreauxi (Fitzgibbon et al. 2017), we are not able to draw conclusions on whether the puerulus stage may be more vulnerable than the juvenile stage due to differences in the methods of the studies and the results. To elaborate, maximum temperatures tested differed $\left(25^{\circ} \mathrm{C}\right.$ compared to $30^{\circ} \mathrm{C}$ in this study), acclimation times differed (60 d compared to 2 days in this study) and the results showed different thermal patterns (exponential increase in aerobic scope to $25^{\circ} \mathrm{C}$ in juveniles compared to a peak in optimal performance at $27^{\circ} \mathrm{C}$ in this study). As there is no comparable study on the thermal tolerance of escape speed of other life stages of this species, we cannot yet judge if this pattern holds true either.

\subsection{Escape speed as an important survival factor}

Escape speed is considered to be an important survival factor in predation interactions, and therefore escape speed performance may be a highly selected trait (Dell et al. 2011). Model results found that the effect of temperature on both maximum and average escape speed was not significant $(\mathrm{p}=$ 0.053 and $p=0.053$ respectively; Fig. 3a, Table 1), which suggests that speed may be relatively unaffected by changes in temperature. This may be due to escape speed being a critical survival measure for a prey species to be able to survival a predator attack (Dell et al. 2011). Other studies investigating the effect of temperature on escape responses have shown mixed results; some found that the temperatures they tested did not affect escape speed (Killen et al. 2015), while others found that temperature had a significant effect on escape speed (Johnson \& Bennett 1995). The difference between escape speed performance suggests that the mechanisms driving how organisms escape from predators varies between species and may be influenced by changing ecosystem pressures throughout different life stages. Escape speed in $S$. verreauxi appears to be unaffected by temperature, suggesting that escape speed during this life stage may be important for survival and therefore strongly selected for.

\subsection{Recovery measures}

Recovery time and EPOC showed different patterns of response to temperature. For time taken to recover to pre-exercise RMR, there was a separation between animals at the higher temperatures that recovered relatively quickly, and those that did not come back to their routine metabolic rate for the remaining duration of the experiment. This separation may indicate that some individuals in the population - those able to recover quickly - may have sufficient phenotypic plasticity to survive and a potentially increased capacity to adapt to future tem- 
peratures (Foo \& Byrne 2016). The longer recovery times were correlated with higher values of EPOC. Longer recovery times have also been associated with higher temperatures in other marine invertebrates (Schalkhausser et al. 2014), as well as with individuals that are more vigilant (Killen et al. 2015). Our results indicate that there may be higher costs associated with higher temperatures, such as longer recovery times which may in turn affect survival. At 26,28 and $30^{\circ} \mathrm{C}$, this cost resulted in death during respirometry trials for some pueruli $(\mathrm{n}=1,1$ and 3 , respectively). As the puerulus stage is a non-feeding stage in the life cycle (Phillips et al. 2006), the energy available to them is limited until they moult into their juvenile feeding stage. Thus, this stage is considered to be a potential bottleneck for the population in terms of recruitment success due to high energy demands associated with near-shore settlement while having a fixed energy supply (Fitzgibbon et al. 2014a). Higher future temperatures coupled with predation pressure may have serious consequences for individuals, as they may not be able to recover sufficiently to transition to their next life stage.

\subsection{Ecological implications and potential future changes}

The extension of the East Australian Current in the region (Ridgway 2007) has already facilitated the range extensions of many other species into this area, and further south in Tasmania, including pelagic fish species favoured by fishers such as pink snapper Pagrus auratus (Last et al. 2011) as well as species that have been detrimental to coastal environments such as the long-spine sea urchin Centrostephanus rodgersii (Ling et al. 2009, Johnson et al. 2011). This warming and increase in transport will likely facilitate increased arrival and survival of $S$. verreauxi pueruli in Tasmanian waters in future years with ocean warming. In addition to a possible range extension at the polewards range edge of their distribution, it is also likely that $S$. verreauxi will undergo a range contraction at their equatorward range edge (Fitzgibbon et al. 2014b). As temperatures in the equatorward edge of their range currently reach $28^{\circ} \mathrm{C}$ (Montgomery et al. 2009), with future ocean warming it is likely that ambient temperature will exceed those that are optimal, leading to decreases in performance of puerulus and hence a likely decrease in settlement and survival, resulting in a range contraction at the warmer range edge for the species.

\section{CONCLUSIONS}

The variability in the investigated measures of eastern rock lobster Sagmariasus verreauxi puerulus performance has implications for this species in terms of selection of particular phenotypes (Clark et al. 2017), recruitment success (Fitzgibbon et al. 2014b) and interactions with other species (Dell et al. 2011). While this study considered multiple possible capacities affecting range shifts, future work should include other measures of performance, including growth at different life stages, reproductive capacity, as well as other measures of species interaction success such as competitive ability for resources and performance of predators. It would also be valuable to identify potential life history bottlenecks that may have a disproportionate effect on populations and examine the thermal tolerances of these particular stages. The more performance measures that are investigated, the greater confidence we will have regarding our capacity to predict responses to future change. In addition, investigating the thermal tolerances at the cooler edge of the species' range would be valuable in determining the implications for settlement survival and species success in the cooler waters of Tasmania. Measures of species performance, including those representative of species interactions, will help develop mechanistic models and more robust and accurate future predictions (Evans et al. 2015).

Acknowledgements. We thank the IMAS aquaculture technicians for their laboratory support and supply of cultured lobsters. Thanks to J. Seager and J. Hulls for their stereovideo support, M. Corkill for multiple reviews of the early manuscript and K. Hartmann and S. Wotherspoon for help with statistics. This research was supported by G.T.P.'s ARC Future Fellowship (FT140100596), Q.P.F. and C.G.C.'s ARC Industrial Transformation Research Hub project (IH12100032) and S.T.'s Holsworth Wildlife Research Endowment. We appreciate the comments from the anonymous reviewers and the editor that improved this contribution.

\section{LITERATURE CITED}

Cairns DK, Gaston AJ, Huettmann F (2008) Endothermy, ectothermy and the global structure of marine vertebrate communities. Mar Ecol Prog Ser 356:239-250

Clark TD, Sandblom E, Jutfelt F (2013) Aerobic scope measurements of fishes in an era of climate change: respirometry, relevance and recommendations. J Exp Biol 216: 2771-2782

Clark TD, Messmer V, Tobin AJ, Hoey AS, Pratchett MS (2017) Rising temperatures may drive fishing-induced selection of low-performance phenotypes. Sci Rep 7: 40571 
Dell AI, Pawar S, Savage VM (2011) Systematic variation in the temperature dependence of physiological and ecological traits. Proc Natl Acad Sci USA 108:10591-10596

Dell AI, Pawar S, Savage VM (2014) Temperature dependence of trophic interactions are driven by asymmetry of species responses and foraging strategy. J Anim Ecol 83: $70-84$

Donelson JM, McCormick MI, Booth DJ, Munday PL (2014) Reproductive acclimation to increased water temperature in a tropical reef fish. PLOS ONE 9:e97223

Doney SC, Ruckelshaus M, Emmett Duffy J, Barry JP and others (2012) Climate change impacts on marine ecosystems. Annu Rev Mar Sci 4:11-37

Eme J, Bennett WA (2009) Acute temperature quotient responses of fishes reflect their divergent thermal habitats in the Banda Sea, Sulawesi, Indonesia. Aust J Zool $57: 357-362$

Emery AR (1973) Comparative ecology and functional osteology of fourteen species of damselfish (Pisces: Pomacentridae) at Alligator Reef, Florida Keys. Bull Mar Sci 23: 649-770

Evans TG, Diamond SE, Kelly MW (2015) Mechanistic species distribution modelling as a link between physiology and conservation. Conserv Physiol 3:cov056

Fitzgibbon QP, Battaglene SC (2012) Effect of photoperiod on the culture of early-stage phyllosoma and metamorphosis of spiny lobster (Sagmariasus verreauxi). Aquaculture 368-369:48-54

Fitzgibbon QP, Battaglene SC, Ritar AJ (2012) Effect of water temperature on the development and energetics of early, mid and late-stage phyllosoma larvae of spiny lobster Sagmariasus verreauxi. Aquaculture 344-349:153-160

Fitzgibbon QP, Jeffs AG, Battaglene SC (2014a) The Achilles heel for spiny lobsters: the energetics of the non-feeding post-larval stage. Fish Fish 15:312-326

Fitzgibbon QP, Ruff N, Tracey SR, Battaglene SC (2014b) Thermal tolerance of the nektonic puerulus stage of spiny lobsters and implications of ocean warming. Mar Ecol Prog Ser 515:173-186

Fitzgibbon QP, Simon CJ, Smith GG, Carter CG, Battaglene SC (2017) Temperature dependent growth, feeding, nutritional condition and aerobic metabolism of juvenile spiny lobster, Sagmariasus verreauxi. Comp Biochem Physiol A Mol Integr Physiol 207:13-20

Foo SA, Byrne M (2016) Acclimatization and adaptive capacity of marine species in a changing ocean. Adv Mar Biol 74:69-116

Grigaltchik VS, Ward AJW, Seebacher F (2012) Thermal acclimation of interactions: differential responses to temperature change alter predator-prey relationship. Proc $\mathrm{R}$ Soc B 279:4058-4064

Hamner WM, Jones MS, Carleton JH, Hauri IR, Williams DM (1988) Zooplankton, planktivorous fish, and water currents on a windward reef face: Great Barrier Reef, Australia. Bull Mar Sci 42:459-479

Harvey E, Shortis M, Stadler M, Cappo M (2002) A Comparison of the accuracy and precision of measurements from single and stereo-video systems. Mar Technol Soc J 36: 38-49

*Hobday A, Pecl G (2014) Identification of global marine hotspots: sentinels for change and vanguards for adaptation action. Rev Fish Biol Fish 24:415-425

Johnson T, Bennett A (1995) The thermal acclimation of burst escape performance in fish: an integrated study of molecular and cellular physiology and organismal per- formance. J Exp Biol 198:2165-2175

Johnson CR, Banks SC, Barrett NS, Cazassus F and others (2011) Climate change cascades: shifts in oceanography, species' ranges and subtidal marine community dynamics in eastern Tasmania. J Exp Mar Biol Ecol 400:17-32

Jutfelt F, Norin T, Ern R, Overgaard J and others (2018) Oxygen- and capacity-limited thermal tolerance: blurring ecology and physiology. J Exp Biol 221:jeb169615

Katersky RS, Carter CG (2007) High growth efficiency occurs over a wide temperature range for juvenile barramundi Lates calcarifer fed a balanced diet. Aquaculture 272:444-450

Killen SS, Reid D, Marras S, Domenici P (2015) The interplay between aerobic metabolism and antipredator performance: vigilance is related to recovery rate after exercise. Front Physiol 6:111

* Kordas RL, Harley CDG, O'Connor MI (2011) Community ecology in a warming world: the influence of temperature on interspecific interactions in marine systems. J Exp Mar Biol Ecol 400:218-226

*Last PR, White WT, Gledhill DC, Hobday AJ, Brown R, Edgar GJ, Pecl G (2011) Long-term shifts in abundance and distribution of a temperate fish fauna: a response to climate change and fishing practices. Glob Ecol Biogeogr 20:58-72

Ling SD, Johnson CR, Ridgway K, Hobday AJ, Haddon M (2009) Climate-driven range extension of a sea urchin: inferring future trends by analysis of recent population dynamics. Glob Change Biol 15:719-731

* Montgomery SS, Liggins GW, Craig JR, McLeod JR (2009) Growth of the spiny lobster Jasus verreauxi (Decapoda: Palinuridae) off the east coast of Australia. NZ J Mar Freshw Res 43:113-123

Norin T, Malte H, Clark TD (2014) Aerobic scope does not predict the performance of a tropical eurythermal fish at elevated temperatures. J Exp Biol 217:244-251

\%̈hlund G, Hedström P, Norman S, Hein CL, Englund G (2015) Temperature dependence of predation depends on the relative performance of predators and prey. Proc $\mathrm{R}$ Soc B 282:20142254

* Paine RT (1974) Intertidal community structure. Oecologia 15:93-120

*Pecl GT, Hobday AJ, Frusher S, Sauer WHH, Bates AE (2014) Ocean warming hotspots provide early warning laboratories for climate change impacts. Rev Fish Biol Fish 24:409-413

Pecl GT, Araújo MB, Bell JD, Blanchard J and others (2017) Biodiversity redistribution under climate change: impacts on ecosystems and human well-being. Science 355: eaai9214

Phillips BF, Booth JD, Cobb JS, Jeffs AG, McWilliam P (2006) Larval and postlarval ecology. In: Phillips BF (ed) Lobsters: biology, management, aquaculture and fisheries. Blackwell Publishing, Oxford, p 231-262

* Pinsky ML, Worm B, Fogarty MJ, Sarmiento JL, Levin SA (2013) Marine taxa track local climate velocities. Science 341:1239-1242

* Pitt NR, Poloczanska ES, Hobday AJ (2010) Climate-driven range changes in Tasmanian intertidal fauna. Mar Freshw Res 61:963-970

* Poloczanska ES, Brown CJ, Sydeman WJ, Kiessling W and others (2013) Global imprint of climate change on marine life. Nat Clim Chang 3:919-925

* Pörtner HO, Farrell AP (2008) Physiology and climate change. Science 322:690-692 
Pörtner HO, Knust R (2007) Climate change affects marine fishes through the oxygen limitation of thermal tolerance. Science 315:95-97

R Core Team (2017) R: a language and environment for statistical computing. R Foundation for Statistical Computing, Vienna

Ridgway KR (2007) Long-term trend and decadal variability of the southward penetration of the East Australian Current. Geophys Res Lett 34:L13613

Robinson L, Gledhill D, Moltschaniwskyj N, Hobday A and others (2015) Rapid assessment of an ocean warming hotspot reveals 'high' confidence in potential species' range extensions. Glob Environ Change 31:28-37

Schalkhausser B, Bock C, Pörtner HO, Lannig G (2014) Escape performance of temperate king scallop, Pecten

Editorial responsibility: Inna Sokolova, Rostock, Germany maximus under ocean warming and acidification. Mar Biol 161:2819-2829

Storch D, Fernández M, Navarrete SA, Pörtner HO (2011) Thermal tolerance of larval stages of the Chilean kelp crab Taliepus dentatus. Mar Ecol Prog Ser 429:157-167

Sunday JM, Bates AE, Dulvy NK (2012) Thermal tolerance and the global redistribution of animals. Nat Clim Chang 2:686-690

* Sunday JM, Pecl GT, Frusher S, Hobday AJ and others (2015) Species traits and climate velocity explain geographic range shifts in an ocean-warming hotspot. Ecol Lett 18:944-953

Watson SA, Morley S, Bates A, Clark M and others (2014) Low global sensitivity of metabolic rate to temperature in calcified marine invertebrates. Oecologia 174:45-54

Submitted: July 18, 2018; Accepted: July 2, 2019

Proofs received from author(s): August 6, 2019 